\section{D software reconstruction for planning robotic assisted radical nephrectomy with level III caval thrombus}

\author{
Marcos Tobias-Machado ${ }^{1,2 *}$, Ricardo JF de Bragança', Rafael \\ Tourinho-Barbosa ${ }^{1}$ Aurus M Dourado ${ }^{3}$ and Hamilton C Zampolli ${ }^{1}$
}

\author{
${ }^{1}$ Instituto do Cancer, Dr Arnaldo Vieira de Carvalho, São Paulo, Brazil \\ ${ }^{2}$ Hospital Sao Luis Morumbi, Rede D'or, São Paulo, Brazil \\ ${ }^{3}$ Hospital São Marcos, Teresina, Piaui, Brazil
}

\section{Introduction}

Inferior vena cava (IVC) involvement by intraluminal extension of tumor is infrequent, occuring in $4 \%$ to $10 \%$ of patients with renal cell carcinoma (RCC) [1-5]. Based on the cephalic extension of the thrombus, Mayo [6] described a classification of inferior vena cava thrombi in 4 categories, which has implications on surgical complexity, estimated blood loss (EBL) and peri-operative complications, but not cancerspecific survival $[2,7]$. Level III IVC thrombus is classified as being located in the retro-hepatic IVC below the diaphragm. Total resection of this tumor is the best chance of cure when no distant metastases are present $[4,8]$. Actually, open radical nephrectomy with concomitant thrombectomy is still the standard treatment. This procedure is technically challenging and involves a large incision and prolonged convalescence [9]. Recently, the feasibility of robotic IVC thrombectomy has been demonstrated, with potential lower EBL and shorter hospitalization and convalescence [7,10-14]. This surgery requires thorough knowledge of surgical anatomy, detailed pre-operative preparation and meticulous robotic technique [7]. The key point in the surgical management is the correct assessment of the extension of the endocaval thrombus, what is mainly based on radiological examinations [8]. Although Ultrasonography (US) and computerized tomography (CT) are useful in demonstrating the extent of the thrombus, CT is not always accurate in delineating the superior margin of the tumor in the IVC. More precisely, magnetic resonance imaging (MRI) can demonstrate a tumor thrombus and its extension, besides signs of wall invasion, being extremely useful to surgical procedure planning $[8,15]$. Vena cavography is not additive to US, CT, and MRI, and it increases the risk of contrast-associated renal injury $[4,8]$. However, new modern

\section{More Information}

*Address for Correspondence: Marcos Tobias-
Machado, MD, Department of Urology of Centro
Universitário Saúde 20 ABC, Av. Lauro Gomes,
2000 - Vila Sacadura Cabral, Santo André, SP,
09060-870, Brazil,
Email: tobias-machado@uol.com.br
Submitted: 03 April 2020
Approved: 29 April 2020
Published: 30 April 2020

How to cite this article: Tobias-Machado M, de Bragança RJF, Tourinho-Barbosa R, Dourado AM, Zampolli HC. 3D software reconstruction for planning robotic assisted radical nephrectomy with level III caval thrombus. Arch Cancer Sci Ther. 2020; 4: 029-033.

DOI: 10.29328/journal.acst.1001019

Copyright: (c) 2020 Tobias-Machado M, et al. This is an open access article distributed under the Creative Commons Attribution License, which permits unrestricted use, distribution, and reproduction in any medium, provided the original work is properly cited.

Check for updates

OPEN ACCESS

image technologies has emerged to help surgical planning, as three-dimensional visualization technique (3DVT) based on routine CT or MRI processed image data [16-20]. Recently, a comparative study showed advantage of 3DVT in management of complex renal tumor during laparoscopic partial nephrectomy [20]. This modality is able to demonstrate anatomy relations, allowing the surgeon to observe the relationship between targeted tumor and peripheral structure before surgery and perform virtual manipulation. This kind of preoperative accurate assessment can enhance surgeons confidence of surgical procedure and decrease surgical risk and incidence of complications [20]. There is no report in the literature of the use of this type of technology in cases of IVC tumor thrombus.

We present the use of 3D holographic interactive reconstruction in a single case of robotic radical nephrectomy with level III IVC thrombectomy.

\section{Methods}

The patient is a 48-year-old male who presented initially with deep venous thrombosis in left leg. No other symptoms. Medical history significant just for obesity. His body mass índex (BMI) was $36,01 \mathrm{Kg} / \mathrm{m} 2$. Lower limbs US showed a left iliac and femoral vein DVT. During investigation, Abdominal 
CT (Figure 1) demostrated a $14 \times 12,4 \times 10,8 \mathrm{~cm}$ right sided renal mass with associated retro-hepatic IVC tumor thrombus. No metastatic evidence. MRI (Figure 2) performed for staging of the thrombus demonstrated a level III tumor thrombus extending into the retro-hepatic IVC, besides deep venous thrombosis in left femoral and iliac vein and IVC below renal vein. Even through MRI, It was difficult the assessment of retro-hepatic thrombus extension, besides renal anatomy (number of renal arteries/veins, collaterals, renal tumor stage), hepatic anatomy (number/location of main hepatic veins, liver involvement) and retroperitoneal anatomy (adenopathy, venous collaterals).

Both CT and MRI were processed into interactive holographic 3D reconstruction by software "Docdo" (Figures $3,4)$. This exam must be ordered through the website www. docdo.com.br. On this website the "DICOM" files of the image exams are sent to the "Docdo" company. Then a team of urologists and radiologists processes the data to a interactive holographic 3D virtual model that can be assessed through the website ou the "Docdo" App. This software allows identify different structures and manipulate the image with enlargement, rotation in several axis and subtraction of structures.

Chest CT for metastatic evaluation was normal Echocardiography with no atrial thrombus. Attempted placement of vena cava filter was unsuccessful (A vena cava filter placement was attempted, but the procedure was aborted because of the poor space between the tumor thrombus and the diaphragm and the risk of filter migration).

After this whole evaluation, we proceeded with robotic radical right nephrectomy with level III IVC thrombectomy.

No angio-embolization was performed neither neoadjuvant therapy.

His preoperative creatinine and hemoglobin levels were $1.1 \mathrm{mg} / \mathrm{dL}$ and $11.5 \mathrm{~g} / \mathrm{dL}$, respectively.
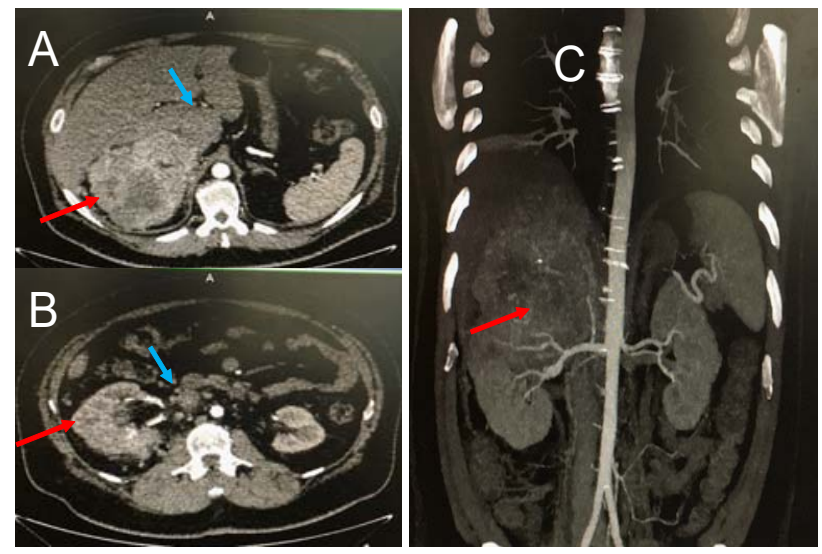

Figure 1: CT scan images (A and B. coronal, C.Sagital) showing a large tumor mass (red arrow) with caval thrombus (blue arrow) identified until infra hepatic region. In this image is not possible to know the exact level of distal extension of caval thrombus.
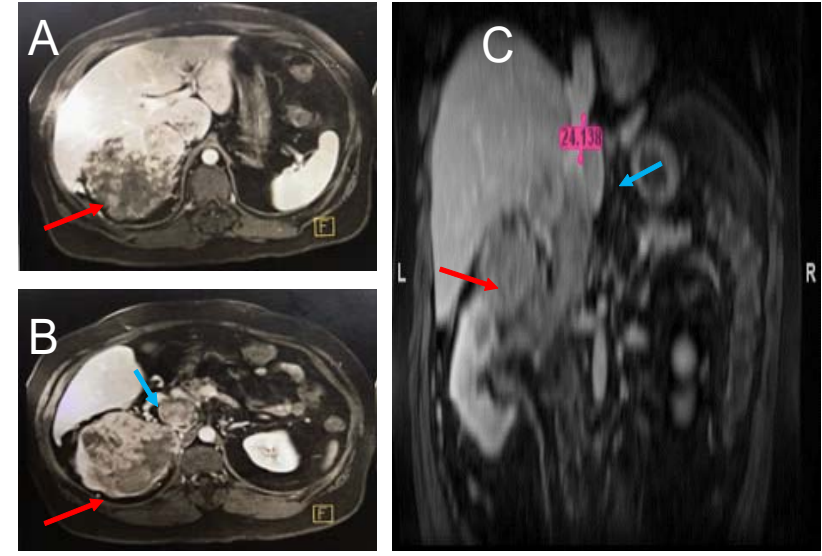

Figure 2: MRI images (A and B. Coronal, C. Sagital) showing a large tumor mass (red arrow) and suggesting a level III caval thrombus (blue arrow) but the distance from the diaphragm and distal portion of thrombus is not well defined.

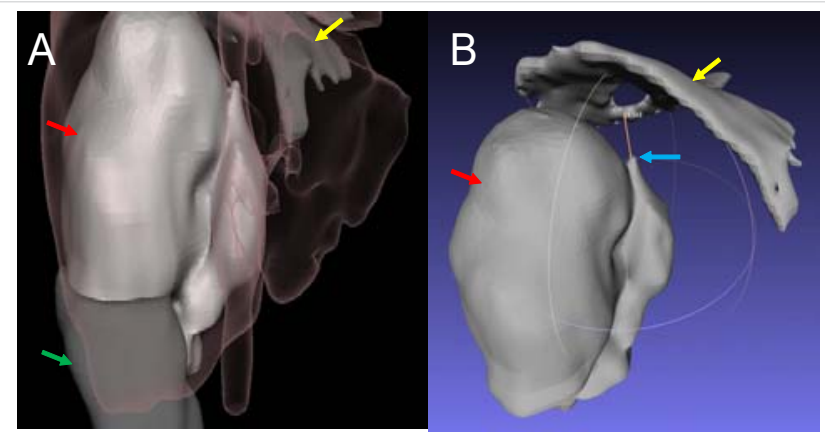

Figure 3: 3D holographic reconstruction: A. Anterior view showing perfectly the relation between renal tumor (red arrow), normal renal parenchyma (green arrow), hepatic border + vena cava (pink shadow) and diafragma (yellow arrow). B. Posterior view showing renal tumor (red arrow), distal extension of thrombus (blue arow) and diaphragm (yellow arrow). There are $3 \mathrm{~cm}$ above the thrombus to put a vessel loop to proximal control.
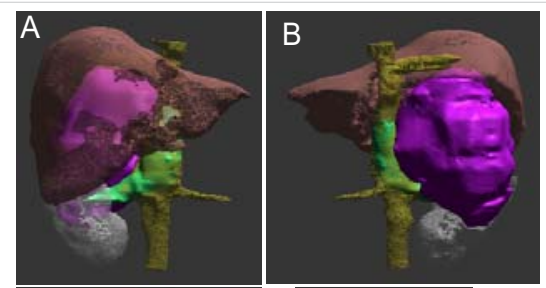

$\square$ Normal renal parenchyma

$\square$ Renal Tumor

$\square$ Vena cava

$\square$ Thrombus

$\square$ Liver
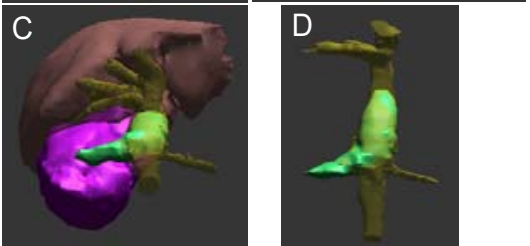

Figure 4: 3D holographic reconstruction: A. anterior view showing perfectly the relation between renal tumor, normal renal parenchyma, liver, veins (renal, hepatic and vena cava) and caval thombus. B. Posterior view showing better the renal tumo and caval thombus. C. topographic anterior view. D. subtracted image showing thrombus in the right renal vein and vena cava up close to the hepatic veins.

\section{Surgical procedure}

The surgery was performed by a experient robotic surgeon with extensive practice in open IVC thrombectomy. Cardiothoracic service was available as needed. The patient was positioned in a modified left lateral decubitus position, with $60^{\circ}$ of table flexion at the level of the anterior superior 
iliac spine. A da Vinci Si robotic platform (Intuitive Surgical, Inc., Sunnyvale, CA) was utilized for this procedure.

The technique used was the same previously described by Gill, et al. ("IVC-first, kidney-last" technique) with a 7-port approach (4 robotic, 2 assistant, 1 liver retraction). We used an intraoperative robotic ultrasound to confirm the thrombus extension, to place the tourniquets in a thrombus-free area and to confirm blood flow cessation.

Real-time intraoperative esophageal ultrasound was not utilized because it was unavailable.

\section{Results}

CT and MRI (2D exams) was able to make the diagnosis of renal tumor and caval thrombus. In addition, they provided information such as tumor size, area of necrosis, relations with adjacent organs in a satisfactory manner. However, they were unable to define the extent of the thrombus and its distance from the diaphragm in this case. Vital information to decide the viability of the surgery and the necessity of thoracic approach and cardiac intervention.

Then 3D CT showed renal and hepatic detailed anatomy, including tumor size, relations, collateral veins, vena cava diameter, tumor thrombus extension and its distance from the diaphragm. The 3D MRI demonstrated thrombus anatomy and retro-hepatic extension, showing a level III tumor thrombus extending into the retro-hepatic IVC, $2 \mathrm{~cm}$ below diaphragm. With this information, we can safely program surgery without the need for a thoracic or cardiac approach.

These data and the quality of the images brought greater accuracy on what to expect during the intraoperative period, extension of the necessary dissection and possible difficulties and risks. In addition, they were very helpful in bringing confidence and some comfort to the surgical team, in the face of such a challenging procedure.

Robotic radical nephrectomy with level III IVC thrombectomy was successfully performed. Total Operative time was 6 hours, 10 minutes. Estimated blood loss was $1800 \mathrm{cc}$. Pacient required transfusion of 4 units of red blood cells intraoperatively. Findings of 3D reconstructions were confirmed by direct vision during dissection (Figure 5) and by robotic US in the intraoperative period.
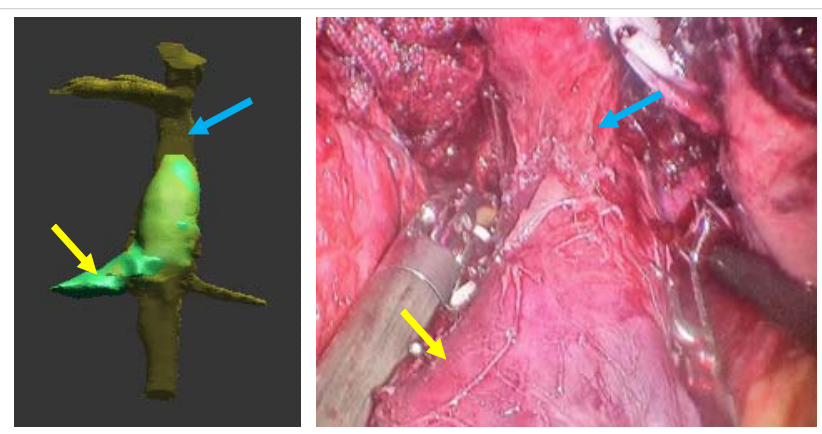

Figure 5: Comparison between 3D reconstruction and intraoperative view showing vena cava (blue arrow) and right renal vein (yellow arrow).
The final pathology reported clear cell renal cell carcinoma (ccRCC), Fuhrman grade 3, pT4N0M0.

\section{Discussion}

Despite the recently demonstrated feasibility of robotic radical nephrectomy with level III IVC thrombectomy, this is still a poorly performed approach because it is a challenging procedure demanding superior surgeon expertise. It can result in considerable blood loss. Inadvertent hemorrhage can occur during dissection of the renal hilum, a region typically containing multiple newly-developed aberrant, large caliber, thin-walled, and high-flow venous collaterals; also, malignant infiltration of hilar and/or peri-caval tissues can lead to considerable oozing, requiring meticulous robotic technique [7]. A good surgical planning can increase the confidence of the surgeon and reduce risk and incidence of complications and may help surgeon to complete surgery successfully [20]. Therefore knowledge of the exact anatomy, locations, distances and diameters of important structures and especially the extension of the endocaval thrombus is essential $[7,8]$. This demand for reducing the difficulty and improvement in surgical safety promotes the emergence and development of medical image technique and device to obtain comprehensive information of anatomical structure in surgical field. Traditional 2D CT has been used to assess factors, including surrounding viscera relationship and vasculature distribution. However, due to its relatively weak capacity of representing intraparenchymal structure 2D CT is difficult to meet surgeons' demand for accurate anatomical information of targeted region. Consequently, a well-established 3D image technology revealing regional surgical anatomy is needed for tumor and vasculature orientation.

Currently, 3D image technology has been applied in multiple fields such as 3D CT, 3D printing, and 3D computed simulation surgery $[16,20]$. Here we described the first experience utilizing a 3D virtual interactive imaging program (Docdo) to plan a robotic Level III IVC thrombectomy. Among "Docdo" advantages are the request through the site which allows the examination to be carried out even at long distances, as it was in our case, and the easy accessibility and handling through the site or the Docdo App. This technology can accurately identify the position of tumor, surrounding vasculature structure, tumor penetration depth, and extension of the endocaval thrombus by anatomy structure tinted by different colors allowing enlargement, rotation on several axis from different angles and subtraction of structures which facilitates surgeons to obtain an unparalleled guidance and understanding for optimization of surgical strategy. In this particular case the 3D CT (Figure 4) was used for renal and hepatic anatomy and 3D MRI (Figure 5) for thrombus anatomy and retro-hepatic extension, showing a level III tumor thrombus extending into the retro-hepatic IVC, $2 \mathrm{~cm}$ below diaphragm what was not clear by the 2D exams. 
Wang, et al. showed that the use of 3D technology in the planning of partial nephrectomy led to a better mean operation time and less complications when compared to traditional CT planning only in complex renal tumors reflecting surgeon's improved comprehensive of procedures and anatomical region. None improvement was achieved for less complex tumors. Also in partial nephrectomy, Shao, et al. reported a 3D vascular model provided effective guidance for precise segmental renal artery clamping, leading to optimal surgical procedure and outcome.

This was our first experience in robotic radical nephrectomy with level III IVC thrombectomy. The total operative time was $6 \mathrm{hrs}, 10$ minutes. Estimated blood loss was $1800 \mathrm{cc}$ and 4 units of red blood cells was transfused intraoperatively.

Mayo Clinic reported their 30-year experience (19702000 ) with open surgical IVC thrombectomy in 540 patients, but only $5 \%(n=28)$ had level III thrombi. Mean operative time was $4.6 \mathrm{hrs}$ (3-8.3), blood loss was 2.7 liters and the majority of patients required blood transfusions, mean 9 units/patient $[2,7,21]$. The University of Southern California revealed 43 patients undergoing open surgical level III thrombectomy over a 32-year period (1978-2012). Mean operative time was $6.1 \mathrm{hrs}$, mean 14 units of blood were transfused per patient $[7,22]$. Gill, et al. report an encouraging initial experience of robotic IVC level III thrombectomy with a mean operative time and blood loss of $4.9 \mathrm{hrs}$ and $375 \mathrm{cc}$, respectively. More similar to us, Ramirez, et al. [23] reported a single case in which he doubled the open technique. Mean operative time was $5 \mathrm{hrs}, 53$ minutes and EBL was $150 \mathrm{cc}$. Apparently there was no improvement in surgical time or blood loss with the use of 3D technology for surgical planning. However, this was our first case and we obtained results similar to those of recent literature. The main contribution of 3D technology in this case was the pre-operative preparation allowing the indication with greater security of robotic approach instead of the open path.

\section{Conclusion}

Robotic surgery for radical nephrectomy with level III IVC thrombectomy is feasible in select patients. At high-volume institutions, robotic surgery appears to be a viable option and with potential lower EBL and transfusion rates. Despite 3D technology and conventional CT presents similar outcomes overall, 3D offers precise visualization of anatomical structure. This may play an important role in aiding surgeon to complete surgery successfully. A larger and well-designed study is needed to evaluate the efficacy, safety and improvements of 3D technology.

\section{References}

1. Marshall FF, Dietrick DD, Baumgartner WA, Reitz BA. Surgica management of renal cell carcinoma with intracaval neoplastic extension above the hepatic veins. J Urol. 1988; 139: 1166.

PubMed: https://www.ncbi.nlm.nih.gov/pubmed/3373579
2. Blute ML, Leibovich BC, Lohse CM, Cheville JC, Zincke H. The Mayo Clinic experience with surgical management, complications and outcome for patients with renal cell carcinoma and venous tumour thrombus. BJU Int. 2004; 94: 33-41.

PubMed: https://www.ncbi.nlm.nih.gov/pubmed/15217427

3. O'Donohoe MK, Flanagan F, Fitzpatrick JM, Smith JM. Surgical approach to inferior vena caval extension of renal cell carcinoma. $\mathrm{Br} \mathrm{J}$ Urol. 1987; 60: 492-496.

4. Hatcher PA, Anderson EE, Paulson DF, Carson CC, Robertson JE. Surgical management and prognosis of renal cell carcinoma invading the vena cava. J Urol. 1991; 145: $20-24$

PubMed: https://www.ncbi.nlm.nih.gov/pubmed/1984092

5. Al Otaibi M, Abou Youssif T, Alkhaldi A, Sircar K, Kassouf W, et a. Renal cell carcinoma with inferior vena caval extension: impact of tumour extent on surgical outcome. BJU Int. 2009; 104: 1467-1470. PubMed: https://www.ncbi.nlm.nih.gov/pubmed/19388993

6. Neves RJ, Zincke H. Surgical treatment of renal cancer with vena cava extension. Br J Urol. 1987; 59: 390-395.

PubMed: https://www.ncbi.nlm.nih.gov/pubmed/3594097

7. Gill IS, Metcalfe C, Abreu A, Duddalwar V, Chopra S, et al. Robotic Level III Inferior Vena Cava Tumor Thrombectomy: Initial Series. J Urol. 194: 929-938.

PubMed: https://www.ncbi.nlm.nih.gov/pubmed/25858419

8. Kaplan S, Ekici S, Dogan R, Demircin M, Özen H, et al. Surgical management of renal cell carcinoma with inferior vena cava tumor thrombus. Am J Surg.2002; 183: 292-299.

PubMed: https://www.ncbi.nlm.nih.gov/pubmed/11943130

9. Varkarakis IM, Bhayani SB, Allaf ME, Inagaki T, Gonzalgo ML, et al. Laparoscopic-assisted nephrectomy with inferior vena cava tumor thrombectomy: preliminary results. Urology. 2004; 64: 925-929. PubMed: https://www.ncbi.nlm.nih.gov/pubmed/15533479

10. Bratslavsky G, Cheng JS. The First Report of Robotic Assisted Radical Nephrectomy with Retrohepatic Vena Caval Tumor Thrombectomy (Level III) Combined with Extended Retroperitoneal Lymph Node Dissection. Urology. 2015; 86: 1235-1240.

PubMed: https://www.ncbi.nlm.nih.gov/pubmed/26254172

11. Abaza R. Initial series of robotic radical nephrectomy with vena caval tumor thrombectomy. Eur Urol. 2011; 59: 652-656. PubMed: https:// www.ncbi.nlm.nih.gov/pubmed/20846783

12. Abaza R, Shabsigh A, Castle E, Allaf M, Hu JC, et al. Multi-Institutional Experience with Robotic Nephrectomy with Inferior Vena Cava Tumor Thrombectomy. J Urol. 2016; 195: 865-871.

PubMed: https://www.ncbi.nlm.nih.gov/pubmed/26602891

13. Wang B, Li H, Ma X, Zhang X, Gu L, et al. Robot-assisted Laparoscopic Inferior Vena Cava Thrombectomy: Different Sides Require Different Techniques. Eur Urol. 2016; 69: 1112-1119.

PubMed: https://www.ncbi.nlm.nih.gov/pubmed/26706105

14. Chopra S, Simone G, Metcalfe C, de Castro Abreu AL, Nabhani J, et al. Robot-assisted Level II-III Inferior Vena Cava Tumor Thrombectomy: Step-by-Step Technique and 1-Year Outcomes. Eur Urol. 2017; 72: 267-274.

PubMed: https://www.ncbi.nlm.nih.gov/pubmed/27663048

15. Myneni L, Hricak H, Carroll PR. Magnetic resonance imaging of renal carcinoma with extension into the vena cava: staging, occuracy and recent advances. Br J Urol. 1991; 68: 571-578.

PubMed: https://www.ncbi.nlm.nih.gov/pubmed/1773285

16. Wang D, Zhang B, Yuan X, Zhang X, Liu C. Preoperative planning and real-time assisted navigation by three- dimensional individual digital model in partial nephrec- tomy with three-dimensional laparoscopic system. Int J Comput Assist Radiol Surg. 2015; 10: 1461-1468. PubMed: https://www.ncbi.nlm.nih.gov/pubmed/25577366 
17. Komai Y, Sakai Y, Gotohda N, Kobayashi T, Kawakami S, et al. A novel 3-dimensional image analysis system for case-specific kidney anatomy and surgical simulation to facilitate clampless partial nephrectomy. Urology. 2014; 83: 500-506.

PubMed: https://www.ncbi.nlm.nih.gov/pubmed/24468517

18. Chen Y, Li H, Wu D, Bi K, Liu C. Surgical planning and manual image fusion based on 3D model facilitate laparoscopic partial nephrectomy for intrarenal tumors. World J Urol. 2014; 32: 1493-1499.

PubMed: https://www.ncbi.nlm.nih.gov/pubmed/24337151

19. Lasser MS, Doscher M, Keehn A, Chernyak V, Garfein E, et al. Virtual surgical planning: A novel aid to robot-assisted laparoscopic partial nephrectomy. J Endourol. 2012; 26: 1372-1379.

PubMed: https://www.ncbi.nlm.nih.gov/pubmed/22642371

20. Wang Z, Qi L, Yuan P, Zu X, Chen W, et al. Application of Theedimensional visualization technology in laparoscopi partial nephrectomy of renal tumor: A comparative study. J Laparoendosc Adv Surg Tech
A. 2017: 27: 516-523.

PubMed: https://www.ncbi.nlm.nih.gov/pubmed/28186431

21. Kearnes RJ, Blute ML. Surgery insight: management of renal cell carcinoma with associated inferior vena cava thrombus. Nat Clin Pract Urol. 2008; 5: 329-339.

PubMed: https://www.ncbi.nlm.nih.gov/pubmed/18477994

22. Patil MB, Montez J, Loh-Doyle J, Cai J, Skinner EC, et al. Level IIIIV inferior vena caval thrombectomy without cardiopulmonary bypass: Long-term experience with intrapericardial control. J Urol. 2014; 192 : 682-689.

PubMed: https://www.ncbi.nlm.nih.gov/pubmed/24704114

23. Ramirez D, Maurice MJ, Cohen B, Krishnamurthi V, Haber GP. Robotic level III IVC tumor thrombectomy: duplicating the open approach. Urology. 2016; 90: 204-207.

PubMed: https://www.ncbi.nlm.nih.gov/pubmed/26802799 